PITHA 05/13, IFIC/05-42,

ZU-TH 17/05, UCLA/05/TEP/27

\title{
QCD Corrections to Static Heavy Quark Form Factors
}

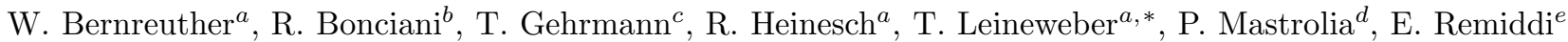 \\ ${ }^{a}$ Institut für Theoretische Physik, RWTH Aachen, D-52056 Aachen, Germany \\ ${ }^{b}$ Departament de Fúsica Tè̀rica, IFIC, CSIC - Universitat de València, E-46071 València, Spain \\ ${ }^{c}$ Institut für Theoretische Physik, Universität Zürich, CH-8057 Zürich, Switzerland \\ ${ }^{d}$ Department of Physics and Astronomy, UCLA, Los Angeles, CA 90095-1547, U.S.A. \\ e Dipartimento di Fisica dell'Università di Bologna and INFN, Sezione di Bologna, I-40126 Bologna, Italy
}

(Dated: April 29, 2019)

\begin{abstract}
Interactions of heavy quarks, in particular of top quarks, with electroweak gauge bosons are expected to be very sensitive to new physics effects related to electroweak symmetry breaking. These interactions are described by the so-called static form factors, which include anomalous magnetic moments and the effective weak charges. We compute the second-order QCD corrections to these static form factors, which turn out to be sizeable and need to be taken into account in searches for new anomalous coupling effects.
\end{abstract}

PACS numbers: 12.15.Mm, 12.38.Bx, 14.65.Fy, 14.65.Ha

Keywords: Top and bottom quarks, Anomalous couplings, Anomalous magnetic moments, QCD corrections

The top quark, so far the heaviest known fundamental particle, will serve as an excellent probe of the fundamental interactions at energies of a few hundred $\mathrm{GeV}$, once more and more top quark events will be accumulated at the Tevatron and, especially, after the CERN Large Hadron Collider (LHC) will have started operating. Even more detailed investigations into top quark properties will be possible at a future linear electronpositron collider (ILC), where top quark pairs can be studied in a very clean and well-defined environment.

In view of its large mass the top quark is, in particular, a unique probe of the dynamics that breaks the electroweak gauge symmetry. If this mechanism differs from the Higgs mechanism of the standard model (SM) of particle physics, observable effects could be found first in top quark production and decay. They may manifest themselves as deviations of the top-quark gauge-boson couplings from the values predicted by the SM (c.f. [1, 2] for overviews).

There has been an enormous effort in recent years to investigate the potential of top quarks, and also of bottom quarks, to new physics effects. Specifically, the couplings to photons and $Z$ bosons, which are the subject of this Letter, have been studied in detail - both for heavy quark production at hadron colliders [ $\underline{3}, 4]$ and at a future high-luminosity high-energetic linear electronpositron collider [5] (for reviews, see [6, 7] ). Indirect constraints, which are rather tight for $b$ quarks, on anomalous contributions to these couplings can be obtained also from electroweak precision observables measured at LEP [3, 8, 9, 10]. Many of these studies attempt to use a "model-independent" approach in that the effect of new interactions is described in terms of effective Lagrangians

*Now at: Framatome ANP GmbH, D-91050 Erlangen, Germany. or, equivalently, in terms of anomalous couplings of heavy quarks, in particular to the SM gauge bosons. In this context the obvious question arises about the size of these effective couplings within the SM, in order to assess the margin of detectability of truly anomalous new physics effects, given the experimental sensitivity of some observable.

In this Letter we investigate the couplings of heavy quarks, noteably of the $t$ and $b$ quark, to the photon and $Z$ boson in higher order QCD. In view of the asymptotic freedom property of the strong interactions and of the large energy scale set by the heavy quark mass, $m_{Q} \gg \Lambda_{Q C D}$, these effective couplings can be computed perturbatively. These couplings do in general depend on the precise kinematics at the vertex, which is expressed in terms of the so-called vertex form factors, depending on the momentum transfer at the vertex. In many applications, it is justified to approximate these form factors by their limits at zero momentum transfer, the so-called static form factors. The most prominent static form factor is the electromagnetic spin-flipping form factor: the anomalous magnetic moment. At present, radiative corrections to the static form factors of quarks are known to one loop in the SM 11, 12], while pure QED corrections to the anomalous magnetic moment are known analytically to three loops [13]. As far as these quantities are concerned, the largest radiative corrections are often those due to QCD. Here we present analytic results for the static $\gamma Q Q$ and $Z Q Q$ form factors to second order in the QCD coupling $\alpha_{s}$ and we predict the size of these moments for $t$ and $b$ quarks within the SM. Moreover we briefly discuss the implications of our results in view of an existing upper bound on the anomalous magnetic moment of the $b$ quark and on future precision measurements of the $\gamma t t$ and $Z t t$ couplings.

To start with we define the static form factors of a heavy quark $Q$ by considering for definiteness the kine- 
matical situation $V^{*} \rightarrow Q\left(p_{1}\right)+\bar{Q}\left(p_{2}\right)\left(V^{*}=\right.$ off-shell photon or $Z$ boson). We study the $V^{*} Q \bar{Q}$ vertex functions $\bar{u}\left(p_{1}\right) \Gamma_{Q}^{\mu, V}(q) v\left(p_{2}\right)$ for on-shell external quarks in the limit of zero four-momentum transfer $q=p_{1}+p_{2}$. In general this vertex function can be decomposed, using Lorentz covariance, into six form factors, two of which are $\mathrm{CP}$-violating. $\mathrm{CP}$ violation in a flavour-conserving vertex function is, in the SM, a tiny, higher-loop induced effect, and we do not consider it here any further. Assuming CP invariance $\Gamma_{Q}^{\mu, V}$ then depends on four form factors:

$$
\begin{aligned}
i \Gamma_{Q}^{\mu, V}= & e\left(F_{1, Q}^{V} \gamma^{\mu}+\frac{i}{2 m_{Q}} F_{2, Q}^{V} \sigma^{\mu \nu} q_{\nu}\right. \\
& \left.+G_{1, Q}^{V} \gamma^{\mu} \gamma_{5}+\frac{1}{2 m_{Q}} G_{2, Q}^{V} \gamma_{5} q^{\mu}\right),
\end{aligned}
$$

where the form factors are functions of $s=q^{2}$. Further $\sigma^{\mu \nu}=\frac{i}{2}\left[\gamma^{\mu}, \gamma^{\nu}\right]$, and $e>0$ denotes the positron charge. If one considers the matrix element of the electromagnetic current, the parity-violating part of Eq. (1) is to be replaced by $G_{Q}\left(\gamma_{\mu} \gamma_{5} s-2 m_{Q} \gamma_{5} q_{\mu}\right)$, where $G_{Q}(0)$ yields the anapole moment of $Q$.

We recall that for a reaction involving the $V Q Q$ vertices the physical object is the $S$ matrix element, but not, in general, these form factors. The static quantities $F_{i, Q}^{\gamma}(0), G_{i, Q}^{\gamma}(0)$ do have a physical meaning: they are defineable as the residues of the photon pole in scattering amplitudes in the soft photon limit, and they are gauge invariant (with respect to the full SM gauge group) and infrared-finite. Likewise, the $F_{i, Q}^{Z}\left(m_{Z}^{2}\right), G_{i, Q}^{Z}\left(m_{Z}^{2}\right)$, which determine the $S$ matrix element of the decay of an on-shell $Z$ boson into a $Q \bar{Q}(Q=b, c)$ quark pair, are gauge invariant - but they are not infrared-finite, as the $Q \bar{Q}$ state above threshold is degenerate with states containing in addition soft photons/gluons and/or collinear massless partons. On the other hand, the static form factors $F_{i, Q}^{Z}(0), G_{i, Q}^{Z}(0)$ are infrared-finite, but gauge invariant only with respect to $\mathrm{QCD}$ or pure QED.

Here our primary aim is to compute the anomalous magnetic moments of heavy quarks in $\mathrm{QCD}$, which are physical quantities. In addition we determine the heavyquark anomalous weak magnetic moment and its axial charge to second order in $\alpha_{s}$. Although these are well-defined objects in QCD only, these SM predictions should serve as useful reference values, in particular for new physics models whose effect on, for instance, the $e^{+} e^{-} \rightarrow Q \bar{Q}$ amplitude is essentially confined to the $V Q \bar{Q}$ vertices.

To lowest order in the SM couplings, only $F_{1, Q}^{\gamma, Z}$ and $G_{1, Q}^{Z}$ are non-zero. The other form factors are generated by one-loop radiative corrections: $G_{1,2, Q}^{\gamma}$ by parityviolating weak corrections, and $F_{2, Q}^{\gamma, Z}$ and $G_{2, Q}^{Z}$ by strong and electroweak corrections. In this Letter we consider the QCD corrections to these form factors. To lowest order in the electroweak couplings we have $G_{1,2, Q}^{\gamma}=0$ and $F_{i, Q}^{V}=v_{Q}^{V} F_{i, Q}(i=1,2)$, where $v_{Q}^{\gamma}=Q_{Q}$ and
$v_{Q}^{Z}=\left(T_{3}^{Q} / 2-s_{w}^{2} Q_{Q}\right) /\left(s_{w} c_{w}\right), a_{Q}=-T_{3}^{Q} /\left(2 s_{w} c_{w}\right)$ are the SM vector and axial vector couplings of $Q$ to the $Z$ boson and the photon in units of $e$, where $s_{w}\left(c_{w}\right)$ is the sine (cosine) of the weak mixing angle, $T_{3}^{Q}$ the third component of the weak isospin and $Q_{Q}$ is the charge of the heavy quark.

Here we determine these form factors in the static limit to second order in $\alpha_{s}$, using the results for non-zero momentum transfer $s$ given in 14]. We work in QCD with $N_{l}$ massless quarks and one quark $Q$ with mass $m_{Q}$ (defined in the on-shell scheme). This includes the case of six quarks with all quarks but the top quark taken to be massless. The QCD coupling $\alpha_{s}=\alpha_{s}(\mu)$ is defined in the standard $\overline{\mathrm{MS}}$ scheme in $N_{f}=N_{l}+1$ flavor QCD with $\mu$ being the renormalization scale.

Because of conservation of the electromagnetic and the neutral vector current, the QCD corrections to $F_{1, Q}$ vanish for $s \rightarrow 0$; i.e., $F_{1, Q}(s=0)=1$. The form factors $F_{2, Q}, G_{1, Q}$, and $G_{2, Q}$ become infrared finite in the static limit. We obtain

$$
F_{2, Q}(s=0)=\frac{\alpha_{s}}{2 \pi} C_{F}+\left(\frac{\alpha_{s}}{2 \pi}\right)^{2} F_{2, Q}^{(2 l)},
$$

with

$$
\begin{gathered}
F_{2, Q}^{(2 l)}=C_{F}^{2}\left(-\frac{31}{4}+2 \zeta_{2}(5-6 \ln (2))+3 \zeta_{3}\right) \\
+C_{F} C_{A}\left(\frac{317}{36}+3 \zeta_{2}(-1+2 \ln (2))-\frac{3}{2} \zeta_{3}\right) \\
+C_{F} T_{F}\left(\frac{119}{9}-8 \zeta_{2}\right)-\frac{25}{9} C_{F} T_{F} N_{l}+C_{F} \beta_{0} \ln \left(r_{Q}\right),(3)
\end{gathered}
$$

where $r_{Q}=\mu^{2} / m_{Q}^{2}, \zeta_{n}$ is the Riemann zeta function, and $C_{F}=\left(N_{c}^{2}-1\right) / 2 N_{c}, C_{A}=N_{c}, T_{F}=1 / 2$ with $N_{c}=$ 3 being the number of colors. Further $\beta_{0}=\left(11 C_{A}-\right.$ $\left.4 T_{F}\left(N_{l}+1\right)\right) / 6$. For $G_{1, Q}$ we have, to order $\alpha_{s}^{2}$ :

$$
G_{1, Q}^{Z}=a_{Q}\left(G_{1, Q}^{(A)}+G_{1, Q}^{(B)}\right)
$$

where the superscripts $A, B$ denote universal and nonuniversal corrections, respectively. We get for the type A term:

$$
G_{1, Q}^{(A)}(s=0)=1-\frac{\alpha_{s}}{2 \pi} C_{F}+\left(\frac{\alpha_{s}}{2 \pi}\right)^{2} G_{1, Q}^{(2 l)},
$$

with

$$
\begin{array}{r}
G_{1, Q}^{(2 l)}=C_{F}^{2}\left(-\frac{29}{12}+8 \zeta_{2}(1-\ln (2))+2 \zeta_{3}\right) \\
+C_{F} C_{A}\left(-\frac{143}{36}+2 \zeta_{2}(-1+2 \ln (2))-\zeta_{3}\right) \\
+C_{F} T_{F}\left(\frac{115}{9}-8 \zeta_{2}\right)+\frac{7}{9} C_{F} T_{F} N_{l}-C_{F} \beta_{0} \ln \left(r_{Q}\right) .
\end{array}
$$

The term $G_{1, Q}^{(B)}$ is obtained by summing over all weak isospin quark doublets in the second order triangle diagram contributions to the $Z Q Q$ vertex [14]. For definiteness we consider here only the static $t$ and $b$ quark form 


\begin{tabular}{ccccc}
\hline \hline & $t$ quark & \multicolumn{3}{c}{$b$ quark } \\
\hline$m_{Q}$ & $175 \mathrm{GeV}$ & & $5 \mathrm{GeV}$ & \\
$Q_{Q}$ & $2 / 3$ & & $-1 / 3$ & \\
$T_{Q}^{3}$ & $1 / 2$ & $m_{b}$ & $-1 / 2$ & \\
\hline$\mu$ & $m_{t}$ & 0.2145 & $m_{Z}$ \\
$\alpha_{s}(\mu)$ & 0.1080 & 0.1187 \\
\hline \hline
\end{tabular}

TABLE I: Input values used in computing the static top and bottom form factors. The mass of the $Z$ boson and the weak mixing angle are taken to be $m_{Z}=91.187 \mathrm{GeV}$ and $\sin ^{2} \theta_{W}=0.231$ [16]. $\alpha_{s}\left(m_{Z}\right)$ is the $\overline{\mathrm{MS}}$ QCD coupling for $N_{f}=5$ flavors, taken from [16], while $\alpha_{s}\left(m_{t}\right)$ is the coupling for $N_{f}=6$ flavors, obtained from $\alpha_{s}\left(m_{Z}\right)$ by renormalizationgroup evolution.

\begin{tabular}{cccc}
\hline \hline & $t\left(\mu=m_{t}\right)$ & $b\left(\mu=m_{b}\right)$ & $b\left(\mu=m_{Z}\right)$ \\
\hline$(g-2)_{Q}^{\gamma,(1 l)} / 2$ & $1.53 \cdot 10^{-2}$ & $-1.52 \cdot 10^{-2}$ & $-8.4 \cdot 10^{-3}$ \\
$(g-2)_{Q}^{\gamma,(2 l)} / 2$ & $4.7 \cdot 10^{-3}$ & $-1.00 \cdot 10^{-2}$ & $-6.6 \cdot 10^{-3}$ \\
\hline$(g-2)_{Q}^{\gamma} / 2$ & $2.00 \cdot 10^{-2}$ & $-2.52 \cdot 10^{-2}$ & $-1.50 \cdot 10^{-2}$ \\
\hline$(g-2)_{Q}^{Z,(1 l)} / 2$ & $5.2 \cdot 10^{-3}$ & $-1.87 \cdot 10^{-2}$ & $-1.03 \cdot 10^{-2}$ \\
$(g-2)_{Q}^{Z,(2 l)} / 2$ & $1.6 \cdot 10^{-3}$ & $-1.24 \cdot 10^{-2}$ & $-8.1 \cdot 10^{-3}$ \\
\hline$(g-2)_{Q}^{Z} / 2$ & $6.8 \cdot 10^{-3}$ & $-3.11 \cdot 10^{-2}$ & $-1.85 \cdot 10^{-2}$ \\
\hline \hline
\end{tabular}

TABLE II: One- and two-loop QCD contributions, and their sums, to the anomalous magnetic and weak magnetic moments of the top and bottom quark, for different values of the renormalization scale $\mu$.

factors. In determining $G_{1, Q}^{(B)}$ we neglect in both cases the mass of the $b$ quark with respect to that of the $t$ quark in the respective triangle diagram. Then we get:

$$
\begin{aligned}
G_{1, t}^{(B)}(0) & =\left(\frac{\alpha_{s}}{2 \pi}\right)^{2} C_{F} T_{F}\left[\mathcal{G}_{1, m_{t}, m_{t}}(0)-\mathcal{G}_{1,0, m_{t}}(0)\right] \\
G_{1, b}^{(B)}(0) & =\left(\frac{\alpha_{s}}{2 \pi}\right)^{2} C_{F} T_{F}\left[\mathcal{G}_{1, m_{b}, m_{b}}(0)-\mathcal{G}_{1, m_{t}, 0}(0)\right]
\end{aligned}
$$

where

$$
\begin{aligned}
\mathcal{G}_{1, m_{Q}, m_{Q}}(0) & =-\frac{19}{3}+\frac{16}{3} \zeta_{2}-3 \ln \left(r_{Q}\right) \\
\mathcal{G}_{1,0, m_{Q}}(0) & =-7-3 \ln \left(r_{Q}\right) \\
\mathcal{G}_{1, m_{Q}, 0}(0) & =\frac{3}{2}-3 \ln \left(r_{Q}\right) .
\end{aligned}
$$

The induced pseudoscalar form factor $G_{2, Q}(0)$ can also be computed to order $\alpha_{s}^{2}$ from the results of [14]. However, it is absent in Eq. (11) for on-shell photons or $Z$ bosons, and irrelevant for the couplings of heavy quarks to leptons and light quarks via $V$ boson exchange. Thus it seems not to be experimentally accessible in the forseeable future. Therefore we shall not present it here.

Let us now consider the static magnetic and weak magnetic form factor of a quark $Q$. We consider

$$
\left(\frac{g-2}{2}\right)_{Q}^{\gamma, Z} \equiv F_{2, Q}^{\gamma, Z}(0)=v_{Q}^{\gamma, Z} F_{2, Q}(0)
$$

\begin{tabular}{cccc}
\hline \hline & $t\left(\mu=m_{t}\right)$ & $b\left(\mu=m_{b}\right)$ & $b\left(\mu=m_{Z}\right)$ \\
\hline$G_{1, Q}^{(A, 1 l)}$ & $-2.29 \cdot 10^{-2}$ & $-4.55 \cdot 10^{-2}$ & $-2.52 \cdot 10^{-2}$ \\
$G_{1, Q}^{(A, 2 l)}$ & $-1.81 \cdot 10^{-3}$ & $-7.74 \cdot 10^{-3}$ & $-1.30 \cdot 10^{-2}$ \\
$G_{1, Q}^{(B)}$ & $1.86 \cdot 10^{-3}$ & $-1.58 \cdot 10^{-2}$ & $-4.85 \cdot 10^{-3}$ \\
\hline$G_{1, Q}^{Z} / a_{Q}$ & $1-2.29 \cdot 10^{-2}$ & $1-6.90 \cdot 10^{-2}$ & $1-4.31 \cdot 10^{-2}$ \\
\hline \hline
\end{tabular}

TABLE III: One- and two-loop QCD contributions to the static form factor $G_{1, Q}^{Z}$ as defined in Eq. (10) for top and bottom quarks.

which correspond to the anomalous magnetic (MDM) and weak magnetic (WMDM) moments of $Q$. (Notice that in the literature the WMDM is often associated with $F_{2, Q}^{Z}\left(s=m_{Z}^{2}\right)$.) We determine the numerical values of these moments for $t$ and $b$ quarks with the above formulae and the input values given in Table 1 . In the case of $(g-2)_{t}^{\gamma, Z} / 2$ we work in $N_{f}=6$ flavor QCD with all quarks but the top quark taken to be massless, while $(g-2)_{b}^{\gamma, Z} / 2$ is computed in the effective $N_{f}=5$ flavor theory with $m_{i}=0(i=u, d, s, c)$ and $m_{b} \neq 0$. The results are given in Table 2. The two-loop QCD contributions to $(g-2)_{t}^{\gamma, Z} / 2$ and $(g-2)_{b}^{\gamma, Z} / 2$ are sizeable: they are about 30 and 70 percent, respectively, of the order $\alpha_{s}$ moments. In the case of the $b$ quark we have evaluated the static magnetic form factors for two different values of the renormalization scale $\mu$, which would apply to different physical situations, for instance, the leptoproduction of $b \bar{b}$ quark pairs above threshold and at the $Z$ resonance. The dependence on $\mu$, which is mainly due to the dependence of $\alpha_{s}$ on this scale, indicates also the size of the unknown higher-order QCD corrections. As to the SM electroweak one-loop contributions to the (W)MDMs of $b$ and $t$ quarks [12]: for the $b$ quark they are significantly smaller than the QCD-induced moments given in Table 2. The weak contributions to the WMDM and, if the SM Higgs boson is light, to the MDM of the $t$ quark are of the order of a few times $10^{-3}$.

Next we consider the weak axial vector charge $\mathbf{a}_{Q}^{Z}$ of a heavy quark $Q$ defined by

$$
\mathbf{a}_{Q}^{Z} \equiv G_{1, Q}^{Z}(0)
$$

where, to second order in the QCD coupling, $G_{1, Q}^{Z}$ receives the type $\mathrm{A}$ and $\mathrm{B}$ contributions given above. $\mathrm{Nu}-$ merical evaluation of these formulae for $t$ and $b$ quarks gives the values of Table 3 . In the case of the $t$ quark the two-loop type A and B contributions almost cancel, while for the $b$ quark the second order corrections are again sizeable.

What do these numbers tell us? For the $b$ quark an upper bound on its magnetic moment was derived in [17] from an analysis of LEP1 data, which, in our convention, reads $\delta(g-2)_{b}^{\gamma} / 2<1.5 \times 10^{-2}(68 \%$ C.L. $)$. Comparing it with Table 2 we see that the QCD-induced contributions to the $b$ quark magnetic moment saturate this bound, which implies that there is limited room for new physics contributions to this quantity. At a future linear collider 
[6, 7], when operated at the $Z$ resonance, the sensitivity to this variable could be improved substantially, either by global fits or by analyzing appropriate angular distributions in $b \bar{b}$ and $b \bar{b} \gamma$ events.

As to the static form factors of the top quark, no such tight constraints exist so far on possible contributions from new interactions (see [9, 10] and [3] for a review). As emphasized above these quantities are particularly sensitive to the dynamics of electroweak symmetry breaking. For instance, in various models with a strongly coupled symmetry breaking sector one may expect contributions from this sector to the static $t$ quark form factors at the 5 - $10 \%$ level [1, 2, 18]. The QCD-induced anomalous magnetic moment and the QCD corrections to the axial charge of the top quark are of the same order of magnitude. Future colliders have the potential to reach this level of sensitivity. At the LHC the $t t \gamma$ and $t t Z$ couplings can be separately measured in associated $t t \gamma$ and $t t Z$ production, respectively. A detailed study of these reactions showed [3, 4] that at the LHC a statistical sensitivity of about $5 \%$ to the photonic couplings $F_{1,2, t}^{\gamma}, G_{1, t}^{\gamma}$ may eventually be reached, while the sensitivity to the analogous couplings to the $Z$ boson is significantly lower. At a future high luminosity linear $e^{+} e^{-}$collider with polarized beams [19] both the $Z$ and the photonic couplings of the top quark will be measurable with a precision of a few percent [6, 7]. Thus it is mandatory to determine these quantities within the SM as precisely as possible.

In conclusion, we have determined the static form factors of $b$ and $t$ quarks, notably their anomalous magnetic moments and axial charges, to second order in the QCD coupling $\alpha_{s}$. Precise knowledge of these effective couplings to photons and $Z$ bosons within the standard model is mandatory in order to assess the margin of detectablity of new physics contributions and to interpret correctly (future) measurements. For the $b$ quark we have found that the QCD contributions to its anomalous magnetic moment saturate the existing experimental upper bound, which implies that there is not much room for new physics effects on this quantity. The QCD corrections to the static form factors of the top quark are of the same order of magnitude as the precision with which these couplings may eventually be measured at future colliders and must therefore be taken into account in searches for anomalous coupling effects.

Acknowledgement: We thank U. Baur, A. Juste and F. Petriello for useful discussions. This work was supported by Deutsche Forschungsgemeinschaft (DFG), SFB/TR9, by DFG-Graduiertenkolleg RWTH Aachen, by HPRN-CT2002-00311 (EURIDICE), by the Swiss National Science Foundation (SNF) under contract 200021101874, and by the USA DoE under the grant DE-FG0391ER40662, Task J.
[1] H. Murayama and M.E. Peskin, Ann. Rev. Nucl. Part. Sci. 46, 533 (1996) hep-ex/9606003.

[2] C.T. Hill and E.H. Simmons, Phys. Rept. 381, 235 (2003) [Erratum-ibid. 390, 553 (2004)] hep-ph/0203079.

[3] U. Baur, A. Juste, L.H. Orr and D. Rainwater, Phys. Rev. D 71, 054013 (2005) hep-ph/0412021.

[4] U. Baur, M. Buice and L.H. Orr, Phys. Rev. D 64, 094019 (2001) hep-ph/0106341.

[5] G.L. Kane, G.A. Ladinsky and C.P. Yuan, Phys. Rev. D 45, 124 (1992); D. Atwood and A. Soni, Phys. Rev. D 45, 2405 (1992); W. Bernreuther, O. Nachtmann, P. Overmann and T. Schröder, Nucl. Phys. B 388, 53 (1992) [Erratum-ibid. B 406, 516 (1993)]; C.P. Yuan, Phys. Rev. D 45, 782 (1992); G.A. Ladinsky and C.P. Yuan, Phys. Rev. D 49, 4415 (1994) hep-ph/9211272; D.O. Carlson, E. Malkawi and C.P. Yuan, Phys. Lett. B 337, 145 (1994) hep-ph/9405277; B. Grzadkowski and Z. Hioki, Nucl. Phys. B 585, 3 (2000) hep-ph/0004223; Z.H. Lin, T. Han, T. Huang, J.X. Wang and X. Zhang, Phys. Rev. D 65 (2002) 014008 hep-ph/0106344.

[6] J.A. Aguilar-Saavedra et al. [ECFA/DESY LC Physics Working Group Collaboration], hep-ph/0106315

[7] T. Abe et al. [American Linear Collider Working Group], in Proc. of the APS/DPF/DPB Summer Study on the Future of Particle Physics (Snowmass 2001) ed. N. Graf, hep-ex/0106057

[8] G. Altarelli, R. Barbieri and S. Jadach, Nucl. Phys. B 369, 3 (1992) [Erratum-ibid. B 376, 444 (1992)]; G. Altarelli, R. Barbieri and F. Caravaglios, Nucl. Phys.
B 405, 3 (1993).

[9] O.J.P. Eboli, M.C. Gonzalez-Garcia and S.F. Novaes, Phys. Lett. B 415, 75 (1997) hep-ph/9704400.

[10] F. Larios, M.A. Perez and C.P. Yuan, Phys. Lett. B 457, 334 (1999) hep-ph/9903394.

[11] W. Hollik, Fortsch. Phys. 38, 165 (1990); A. Czarnecki, B. Krause and W.J. Marciano, Phys. Rev. Lett. 76, 3267 (1996) hep-ph/9512369.

[12] J. Bernabeu, J. Vidal and G.A. Gonzalez-Sprinberg, Phys. Lett. B 397, 255 (1997) hep-ph/9702222; J. Bernabeu, D. Comelli, L. Lavoura and J.P. Silva, Phys. Rev. D 53, 5222 (1996) hep-ph/9509416.

[13] S. Laporta and E. Remiddi, Phys. Lett. B 379, 283 (1996) hep-ph/9602417.

[14] W. Bernreuther et al., Nucl. Phys. B 706, 245 (2005) [hep-ph/0406046]; 712, 229 (2005) hep-ph/0412259; 723, 91 (2005) hep-ph/0504190.

[15] [LEP Collaborations], hep-ex/0412015.

[16] S. Eidelman et al. [Particle Data Group], Phys. Lett. B 592, 1 (2004).

[17] R. Escribano and E. Masso, Nucl. Phys. B 429, 19 (1994) hep-ph/9403304.

[18] R. D. Peccei and X. Zhang, Nucl. Phys. B 337, 269 (1990); R.S. Chivukula, E.H. Simmons and J. Terning, Phys. Rev. D 53, 5258 (1996) hep-ph/9506427; U. Mahanta, Phys. Rev. D 55, 5848 (1997) hep-ph/9611289; Phys. Rev. D 56, 402 (1997).

[19] G. Moortgat-Pick et al., hep-ph/0507011 